\title{
Malattie dei Reni e delle Vie Urinarie
}

\author{
F.P. Schena, F.P. Selvaggi, L. Gesualdo, M. Battaglia
}

$4^{\mathrm{a}}$ edizione con DVD

La quarta edizione di Malattie dei Reni e delle Vie Urinarie, è stata profondamente revisionata nei contenuti e nella forma, che accoglie in copertina per la prima volta Loreto Gesualdo e Michele Battaglia, può oggi essere considerata un'opera completa, in grado di soddisfare le esigenze di diverse categorie di lettori.

Il volume raccoglie in una dimensione contenuta, ma esaustiva le principali patologie nefrologiche e urologiche che si possono incontrare nella pratica clinica quotidiana.

Lopera, che non è stata solo aggiornata, ma completamente riorganizzata, si rivolge infatti agli studenti delle Lauree Sanitarie, a quelli del corso di laurea in Medicina e Chirurgia, agli Specializzandi e infine, come aggiornamento relativo alla disciplina, ai Medici di Medicina di base. A questo scopo, il testo è stato riorganizzato ricorrendo all'utilizzo di un diverso carattere di stampa.
Gli approfondimenti realizzati per gli Specializzandi si presentano perciò con un corpo più piccolo rispetto al resto del volume.

Questa nuova edizione è stata ulteriormente arricchita da un DVD che contiene materiale multimediale indispensabile per integrare lo studio. Un ricco atlante iconografico consentirà agli Specializzandi un ulteriore approfondimento di alcuni argomenti chiave trattati nei vari capitoli del volume.

Inoltre, coloro che seguiranno gli insegnamenti della specialità in Nefrologia e in Urologia potranno visionare sul DVD anche un'inedita raccolta di filmati relativi a interventi chirurgici e a manovre diagnostiche "invasive" sull'apparato urinario. 